\title{
28 Research Suare \\ Detecting COVID-19 in Chest CT Images Based on Several Pre-Trained Models
}

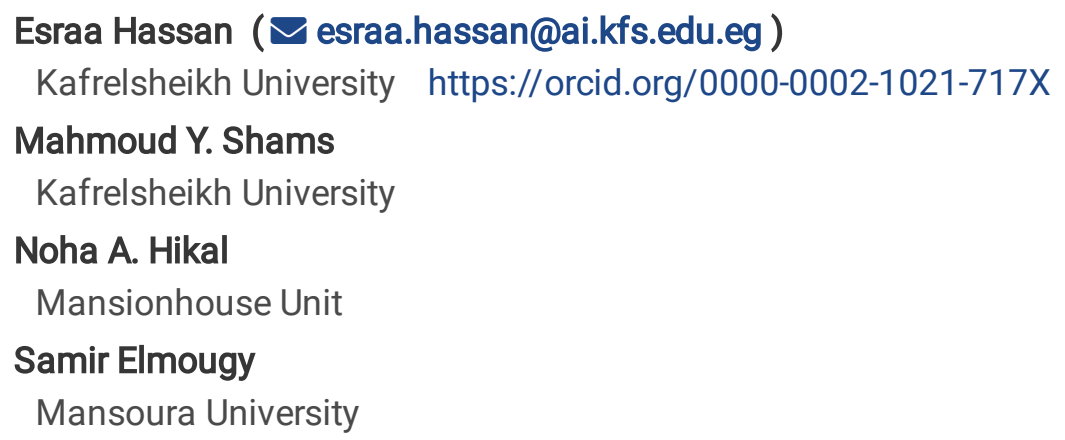

\section{Research}

Keywords: CT scan images, Deep learning, COVID-19, pre-trained models.

Posted Date: January 17th, 2022

DOI: https://doi.org/10.21203/rs.3.rs-1221432/v1

License: (c) (i) This work is licensed under a Creative Commons Attribution 4.0 International License. Read Full License 


\section{Abstract}

COVID-19 virus (coronavirus) is causing a worldwide pandemic of severe respiratory illnesses (SARS-CoV-2). The unique virus was discovered in the Chinese city of Wuhan in December 2019 and has since spread throughout the world. For fear of spreading, the World Health Organization (WHO) issued a Public Health Emergency of International Concern. This paper attempted to offer an autonomous prediction of COVID-19 disease using chest CT scan images by using transfer learning techniques and deep learning algorithms. The dataset included 13413 samples divided into two categories: 7395 CT chest scan images of individuals with confirmed COVID-19 and 6018 images of suspicious cases. The Resnet (50) model has the best training results, Specificity, precision, Negative Predictive Value, False Positive Rate, False Discovery Rate, False Negative Rate, Accuracy, F1 Score, and Matthews Correlation Coefficient with values 0.9880, 0.9892, 0.9891, 0.9882, $0.0108,0.0109,0.0120,0.9886,0.9885$, and 0.9772 respectively.

\section{Introduction}

COVID-19 has infected over 1.3 million people around the world and caused the deaths of over 106,000 cases. Inefficiency and a lack of diagnosis are two major roadblocks to regulating the progression of this disease. Measures using reverse transcription-polymerase chain reaction (RT-PCR) are used in most current assays. It takes 4-6 hours to get results, which is a significant time difference when compared to COVID-19's quick spread rate. As a result, many infected cases go undetected for long periods, infecting others unintentionally. Deep learning (DL) algorithms for diagnosing COVID-19 using CT scans are becoming more popular. As a result, many infected cases go undetected for long periods, infecting others unintentionally [1].

The lack of COVID-19-CT scan images causes a challenge for DL models that have a risk of overfitting when trained on small datasets. We investigate transfer learning to deal with data scarcity. The goal of transfer learning is to use data-rich source tasks to aid learning of a data-deficient target task for a CT-based diagnosis of COVID-19 [2]. We investigate transfer learning to deal with I data scarcity. The goal of transfer learning is to use data-rich source tasks to aid learning of a data-deficient target task for a CT-based diagnosis of COVID-19[3]. Pre-training a powerful visual feature extraction DL models on large images in the source tasks and then fine-tuning the network weights on the target task's tiny dataset is a common strategy. To examine the effects of transfer learning for COVID-19 diagnosis, we develop multiple transferring procedures and conduct detailed analysis in the dimensions of source-target domain difference[4][5]. This paper proposed a PCT model with and support vector machine (SVM) for identifying and forecasting pneumonia from chest CT scan images. Instead of using CNN directly in the classification process, pre-trained models (Inception (v3), Resnet (50), VGG (16), and VGG (19) are used [6]. The binary loss function is used to perform the classification procedure. Transfer learning is used to generate a pneumonia diagnosis model with chest CT scan images. The contributions to this paper are summarised as follows:

To aid in the diagnosis of COVID-19 based on CT scans, we offer a sample-efficient deep learning method.

Using the COVID- 19 CT scan dataset to train and evaluate the CDNN model, which contains 6018 positive CT scans with COVID-19 clinical findings and 7395 negative images.

The rest of the paper is planned as follows. Section 2 presents related works. Section 3 presents methods, and Section 4 experiments. Section 5 concludes the paper.

\section{Related Works}

Researchers have been striving to develop deep learning algorithms for detecting the COVID-19 virus using CT scan images since the outbreak of COVID-19 as shown in Table 1. Cherti et al.[7] perform pre-training and transfer to various natural and medical targets while adjusting network size, source data scale, and domain, which are either huge natural 
ImageNet or large medical chest X-ray datasets[1]. Using a large chest CT data set, Chowdhury et al. [8] suggested an ensemble of deep convolutional neural networks (CNN) based on EfficientNet, dubbed ECOVNet, to detect COVID-19. The open-access large chest X-ray collection is first supplemented, and then ImageNet pre-trained weights for EfficientNet are transferred with some customised fine-tuning top layers that are trained, followed by an ensemble of model snapshots to classify COVID-19, normal, and pneumonia chest X-rays. Osama et al.[9] proposed a model that depends on machine learning algorithms in combatting the virus thus far, focusing on screening, forecasting, and vaccine development. They offer a complete overview of the machine learning algorithms and models that can be utilised on this mission to help combat the infection[10]. Baruch et al.[11] present a strategy for replacing ReLU with a quadratic polynomial activation that is organised. They employ a pre-trained model that trains another HE-friendly model, utilising approaches like "trainable activation" functions and knowledge distillation to address the accuracy degradation issue. They use the chest X-ray and CT datasets to demonstrate their methods using the AlexNet architecture for COVID-19 detection. Hao et al. [12]present a data-efficient classification and active learning architecture. It is based on (1) unsupervised convolutional neural network representation learning and (2) the Gaussian Process approach. Self-supervision is used in unsupervised representation learning, which does not require class labels, and the learned features have been shown to produce a labelefficient classification. They investigated the several pre-trained models to evaluate analytics measures of COVID-19 classification, according to Maheen et al.[13]. Dong et al.[14] introduce DL (RCoNetsk) model that uses Deformable Mutual Information Maximization(DMIM) model, Mixed High-order Moment Feature(MHMF) model, and Multi-Expert Uncertaintyaware Learning(MEUL) model robust COVID-19 In [15] proposed a Mutual Information (MI) between input data and latent representations to be accurately calculated to capture compact and disentangled representational properties[16]. Deep Learning Software Vision(DLSV) model was proposed by Arjun et al.[17] by using X-rays COVIDx cases were classified using DL. The results are compared to COVID-Net and other DL models available in the open-source community. Humans cannot understand how or why a model classifies an image into a certain class; hence, DL technologies are categorizing chest CT scan images into one of three classes, Harkness et al.[18] suggested models are trained using a CT scan dataset and tested on a test set and a hospital dataset. Standard classification metrics are used to assess the models' classification performance, explainability modules are used to investigate the most essential image aspects for categorization[19].

Table 1

Several comparisons between the related works

\begin{tabular}{|c|c|c|c|c|}
\hline Year & Authors & Tasks & Model & Metrics \\
\hline 2021 & Cherti et al.[7] & $\begin{array}{l}\text { Few-Shot Learning Image } \\
\text { Classification Transfer Learning }\end{array}$ & Few-Shot Transfer Learning & $\mathrm{ACC}=78.35 \%$ \\
\hline 2020 & $\begin{array}{l}\text { Chowdhury } \\
\text { et al.[8] }\end{array}$ & Fine-tuning & ECOVNet model & $\begin{array}{l}\text { Precision= } \\
98.95 \%\end{array}$ \\
\hline 2021 & $\begin{array}{l}\text { Baruch et al. } \\
\text { [11] }\end{array}$ & Knowledge Distillation & $\begin{array}{l}\text { Adaptation of the knowledge } \\
\text { distillation }\end{array}$ & $A c c=0.913 \%$ \\
\hline 2021 & Hao et al.[12] & Image Classification & CNN model & $\begin{array}{l}\text { Precision= } \\
95 \%\end{array}$ \\
\hline 2021 & $\begin{array}{l}\text { Maheen et al. } \\
\text { [13] }\end{array}$ & $\begin{array}{l}\text { Image Classification for Computed } \\
\text { Tomography (CT) }\end{array}$ & Pre-trained CNN Model & $A c c=98.33 \%$ \\
\hline 2021 & $\begin{array}{l}\text { Dong et al. } \\
\text { [14] }\end{array}$ & $\begin{array}{l}\text { Image Classification for Computed } \\
\text { Tomography (CT) }\end{array}$ & linear mixed model & $\mathrm{N} / \mathrm{A}$ \\
\hline 2021 & $\begin{array}{l}\text { Harkness et } \\
\text { al.[18] }\end{array}$ & Classification Pneumonia Detection & CNN model & $\mathrm{N} / \mathrm{A}$ \\
\hline 2020 & $\begin{array}{l}\text { Arjun et al. } \\
{[17]}\end{array}$ & COVID-19 Chest X-rays classification & COGNEX model & $P P V=93.3 \%$ \\
\hline
\end{tabular}




\section{The Proposed Model}

Our work depends on using an SVM classifier with different optimizers for the diagnosis of COVID-19. It depends on the idea of transfer learning from pre-trained models such as Inception-V3, Resnet (50), VGG (16), and VGG (19). Used in this work is the COVID-X dataset of chest CT-scan images. As shown in Fig. 1, some preprocessing stages are used followed by extracting the features that are used by SVM for classification tasks based on the cross-entropy loss function. Table 2 shows the training hyperparameters used while fine-tuning the pre-trained model.

\section{Preprocessing And Data Augmentation}

CT images are downsized to $224 \times 224 \times 3$ in size. In some circumstances, getting enough information is challenging, especially in medical cases, because it is a costly and time-consuming process. Data augmentation addresses this issue by maximizing the usage of existing data while also addressing the absence of input data. After the dataset has been preprocessed and partitioned, it is used in the training process to increase training data, reduce the danger of over-fitting, and improve accuracy.

\subsection{Pre-trained CNN architecture for feature extraction}

In medical image processing applications, the DNN suggested model has demonstrated superior performance. Due to the restricted availability of CT chest samples, it will be difficult to train these algorithms from scratch to predict pneumonia. In such situations, the use of pre-trained models based on the transfer learning idea can be advantageous. The transfer learning technique is used to provide a pre-trained structure in an information base that might be from the same or a different domain, allowing users to solve new problems more quickly and effectively. Rather than looking at the traditional process of training with randomly initialized weights, a pre-trained model is used as the starting point for a few specified jobs. As a result, it aids in the reduction of the significant computing resources necessary to create neural network models to address these difficulties. Inception v3, Resnet (50), VGG (16), and VGG (19) are the pre-trained CNN models used in this paper.

\subsection{Classification methods}

After the features were extracted automatically using pre-trained models, two classifiers were used for the classification of chest CT scan images based on the extracted features. 
Table 2

The detailed computations and main values of all CDNN model layers

\begin{tabular}{|c|c|c|}
\hline Name of Layer & Filter Size (FS) and Stride (S) & Activation \\
\hline Input layer & - & $(80,80,3)$ \\
\hline Convolutional layer1 & $\mathrm{FS}=(2,2), \mathrm{S}=1$, Padding=same, filters $=128$ & $(80,80,128)$ \\
\hline Convolutional layer2 & $F S=(2,2), S=1$, Padding=same, filters $=128$ & $(80,80,128)$ \\
\hline Max Pooling & $\mathrm{PS}=(2,2), \mathrm{S}=2$, Padding=valid & $(40,40,128)$ \\
\hline Convolutional layer3 & $F S=(2,2), S=1$, Padding=same, filters $=64$ & $(40,40,64)$ \\
\hline Convolutional layer3 & $\mathrm{FS}=(2,2), S=1$, Padding=same, filters $=64$ & $(40,40,64)$ \\
\hline Batch normalization & Momentum=0.99, Epsilion=0.001, Axis=3 & $(40,40,64)$ \\
\hline Max Pooling & $P S=(2,2), S=2$, Padding=valid & $(20,20,64)$ \\
\hline Convolutional layer3 & $F S=(2,2), S=1$, Padding=same, filters $=32$ & $(20,20,32)$ \\
\hline Convolutional layer3 & $F S=(2,2), S=1$, Padding=same, filters $=32$ & $(20,20,32)$ \\
\hline Convolutional layer3 & $F S=(2,2), S=1$, Padding=same, filters $=32$ & $(20,20,32)$ \\
\hline Convolutional layer3 & $F S=(2,2), S=1$, Padding=same, filters $=32$ & $(20,20,32)$ \\
\hline Max Pooling & $P S=(2,2), S=2$, Padding=valid & $(10,10,32)$ \\
\hline Convolutional layer3 & $F S=(2,2), S=1$, Padding $=$ same, filters $=40$ & $(10,10,40)$ \\
\hline Convolutional layer3 & $F S=(2,2), S=1$, Padding $=$ same, filters $=40$ & $(10,10,40)$ \\
\hline Convolutional layer3 & $F S=(2,2), S=1$, Padding=same, filters $=40$ & $(10,10,40)$ \\
\hline Convolutional layer3 & $F S=(2,2), S=1$, Padding $=$ same, filters $=40$ & $(10,10,40)$ \\
\hline Max Pooling & $P S=(2,2), S=2$, Padding=valid & $(5,5,40)$ \\
\hline Flatten & - & (None,1000) \\
\hline Dense & Units $=2048$, Activation=Linear & (None,2048) \\
\hline Activation & ReLU & (None,2048) \\
\hline Dense & Units $=1024$, Activation=Linear & (None,1024) \\
\hline Batch normalization & Momentum=0.99,Epsilion=0.001,Axis=1 & (None,1024) \\
\hline Activation & ReLU & (None,1024) \\
\hline Dropout & Rate $=0.4$ & (None,1024) \\
\hline Dense & Units=1024 & (None,1024) \\
\hline Activation & ReLU & (None,1024) \\
\hline Dense & Units $=4$, activation $=$ SoftMax & (None,4) \\
\hline Output & & (None,4) \\
\hline
\end{tabular}

\subsection{Binary Crossentropy Classifier}

The loss function binary cross-entropy is utilised in binary classification tasks. On many two-category tasks, it equals the average of the categorical cross-entropy loss that is calculated as: 


$$
\text { loss }=\frac{1}{\text { output size }} \sum_{i=1}^{\text {output size }} y_{i} \log \hat{y}_{i}+\left(1-y_{i}\right) \log \left(1-\hat{y}_{i}\right)
$$

Where $\hat{y}$, is the i-th scalar value in the model output, $y_{i}$ is the corresponding target value, and the output size is the number of scalar values in the model output.

The binary cross-entropy is very convenient to train a model to solve many classification problems at the same time if each classification can be reduced to a binary choice. Table 3 displays the network's hyperparameters. We compare between the Stochastic gradient descent (SGD) and Adaptive Moment Estimation(ADAM) optimizers set the learning rate at 0.001, the momentum at 0.9, and the batch size 64 for SGD and optimizers set the learning rate at 0.001, the momentum at 0.9 , and the batch size 64 and set the learning rate at 0.001 , the momentum at 0.9 , and the batch size 64 for SGD and optimizers set the learning rate at 0.001 Beta1=0.9, Beta2=0.999 for Adam optimizer.

Table 3:Training hyperparameters used while fine-tuning the deep learning model

\begin{tabular}{|c|c|c|}
\hline \multicolumn{3}{|c|}{ Hyperparameters of CDNN model } \\
\hline Optimizer & Adam & SGD \\
\hline Learning rate & 0.001 & 0.001 \\
\hline Batch Size & 32 & 32 \\
\hline No. Epochs & 100 & 100 \\
\hline Beta1 & 0.9 & None \\
\hline Beta2 & 0.999 & None \\
\hline Epsilon & None & None \\
\hline Decay & 0 & 0 \\
\hline Amsgrad & False & False \\
\hline
\end{tabular}

\section{Results And Discussion}

This section discussed includes (i) the experimental setup, (ii) the pre-processing steps, (iii) the used-dataset, (vi) the evaluation measures, ( $v$ ) then the results of our proposed method. To ensure the effectiveness of our proposed model, the performance was compared with the performance of each pre-trained model individually.

\subsection{Experimental setup}

All of the experiments were run on a laptop with a dual 8 GB GPU and 256 GB RAM. The average time of an epoch was multiplied by the number of epochs until the early stopping was used to calculate the training processing time. The remainder of this section is organized as follows. To compare them to well-known network models, we first explain image preprocessing, including contrast stretching and background removal. This inquiry on a deep FV approach is still ongoing. Finally, we show the results of scan-based categorization, which are calculated by combining patch-based scores. We compare the dataset to various pre-trained models for diagnosing cases, which are based on a variety of input data attributes.

\subsection{Pre-processing}

Image augmentation artificially constructs training images using a variety of processing techniques or a mix of techniques, such as random rotation, shifts, shear, and flips, among others[2]. Figure (9) shows the effects of data augmentation for the COVID-19 CT scan image database, which contains data with a wide range of resolution and intensity.

\subsection{Dataset}


COVIDx CT, a benchmark CT image dataset derived from a variety of sources of CT imaging data currently comprising 201,103 images across 4,501 patient cases. Figure (3) shows samples of chest CT images[20].

\subsection{Evaluation Metrics}

The evaluation metrics and CT scan image classification findings are presented in this section. Sensitivity, specificity, Precision, Negative Predictive Value(NPV), False Positive Rate(FPR), False Discovery Rate(FDR), False Negative Rate(FNR), accuracy, F1-score, and Matthews Correlation Coefficient(MCC) were all used to evaluate our model. The number of effectively classified negative and positive cases is defined as TN and TP, respectively. Furthermore, the terms FN and FP refer to the number of misclassified positive and negative instances, respectively. Where These evaluation measures were calculated using the TN, TP, FN, and FP formulas. Table 4 lists the definitions and equations for each evaluation metric. The learning curves are analysis curves that show the evaluation ratio for both training and testing stages. Figure( 4) displays the training accuracy value of the CDNN model and the loss value of the CDNN model in the case SGD optimizer. Figure( 5) displays the training accuracy value of the CDNN model and the Loss value of the CDNN model in the case of Adam optimizer. Figure (6) and Figure (7) display the learning curves for both SGD and Adam optimizers in the testing stage.

Table 4:The Detailed description for measuring evaluation values with equations

\begin{tabular}{|c|c|c|}
\hline Metrics & Description & Equation \\
\hline $\begin{array}{l}\text { Sensitivity } \\
\text { (Recall) }\end{array}$ & The fraction of positive patterne that are correctly classified. & Recall $=\frac{\mathrm{TP}}{(\mathrm{TP}+\mathrm{FN})}$ \\
\hline specificity & A measure of how well a test can identify true negatives. & specificity $=\frac{\mathrm{TN}}{(\mathrm{FP}+\mathrm{TN})}$ \\
\hline Precision & The percentage of retrieved instances that are relevant & Precision $=\frac{T P}{(T P+F P)}$ \\
\hline NPV & The probability that a model with a negative test result is a true negative & $\mathrm{NPV}=\frac{\mathrm{TN}}{(\mathrm{TN}+\mathrm{FN})}$ \\
\hline FPR & The probability of falsely rejecting the null hypothesis for a particular test & $\mathrm{FPR}=\frac{\mathrm{FP}}{(\mathrm{FP}+\mathrm{TN})}$ \\
\hline FDR & $\begin{array}{l}\text { When conducting multiple comparisons, a meane of conceptualizing the rate of } \\
\text { type I errore in null hypothesis testing. }\end{array}$ & $\mathrm{FDR}=\frac{\mathrm{FP}}{(\mathrm{FP}+\mathrm{TP})}$ \\
\hline FNR & $\begin{array}{l}\text { The proportion of significance tests that failed to reject the null hypothesis when } \\
\text { the null hypothesis is indeed falls }\end{array}$ & $\mathrm{FNR}=\frac{\mathrm{FN}}{(\mathrm{FN}+\mathrm{TP})}$ \\
\hline Accuracy & The ratio of correct predicted classes to the total number of samples evaluated & Accuracy $=\frac{T N+T P}{T P+F P+T N+F N}$ \\
\hline F1-8core & The harmonic average between recall and precision rat & $\mathrm{F} 1-\mathrm{score}=\frac{2 \mathrm{TP}}{2 \mathrm{TP}+\mathrm{FP}+\mathrm{FN}}(12)$ \\
\hline MCC & $\begin{array}{l}\text { A metric for evaluating the accuracy of binary and multiclase classifications. It } \\
\text { takes into account true and false positives and negatives, and is widely regarded } \\
\text { ae a fair measure that can be applied even when the classes are of varying sizes. }\end{array}$ & $\begin{array}{c}\mathrm{MCC}= \\
\mathrm{TP} \cdot \mathrm{TN}-\mathrm{FP} \cdot \mathrm{FN} \\
\frac{(13)}{\mathrm{sqrtt}(\mathrm{TP}+\mathrm{PP}) \cdot(\mathrm{TP}+\mathrm{FN}) \cdot(\mathrm{TN}+\mathrm{FP}) \cdot(\mathrm{TN}+\mathrm{FN}))} \\
(13)\end{array}$ \\
\hline
\end{tabular}

\subsection{Confusion matrix and testing accuracy}

The CDNN model's performance metrics are shown in the table below. It achieved the highest percentages for precision, recall, and F1 score metrics, with a percentage of accuracy compared with the model. It indicates how much the model can distinguish between classes. Figures (8) and (9) show the confusion matrix of SGD and Adam optimizer in both training and testing stages. Calculation of the matrices measures is important for comparing between the models. The CDNN model achieves robustness results for CT scan image classification that are illustrated in Table 5 and Table 6. 
Table 5

The overall common metrics for the testing stage of ResNet(50)(training stage)

\begin{tabular}{|c|c|c|c|c|c|c|c|c|c|c|}
\hline \multicolumn{11}{|c|}{ ResNet(50) Training } \\
\hline Metrics & Recall & Specificity & precision & NPV & FPR & FDR & FNR & Accuracy & $\begin{array}{l}\text { F- } \\
\text { score }\end{array}$ & MCC \\
\hline Adam & 0.9903 & 0.9854 & 0.9851 & 0.9905 & 0.0146 & 0.0149 & 0.0097 & 0.9878 & 0.9877 & 0.9757 \\
\hline SGD & 0.9890 & 0.9853 & 0.9850 & 0.9892 & 0.0147 & 0.0150 & 0.0110 & 0.9871 & 0.9870 & 0.9742 \\
\hline
\end{tabular}

Table 6

The overall common metrics for the testing stage of ResNet(50)(testing stage)

\begin{tabular}{|llllllllllll|}
\hline \multicolumn{1}{|l}{ ResNet(50) Testing } & & & & & & & & & \\
\hline Metrics & Recall & Specificity & precision & NPV & FPR & FDR & FNR & Accuracy & $\begin{array}{l}\text { F- } \\
\text { score }\end{array}$ & MCC \\
Adam & 0.9953 & 0.9862 & 0.9859 & 0.9954 & 0.0138 & 0.0141 & 0.0047 & 0.9907 & 0.9906 & 0.9814 \\
\hline SGD & 0.9933 & 0.9860 & 0.9857 & 0.9935 & 0.0140 & 0.0143 & 0.0067 & 0.9896 & 0.9895 & 0.9793 \\
\hline
\end{tabular}

\section{Conclusion}

This paper proposed a CDNN model for COVID-19, pneumonia, and COVID-19 cases based on CT scan images. The SVM employs the binary cross-entropy loss function to reduce the overfitting ratio between the training and testing results. Many accuracy measures (such as specificity, precision, negative predictive value, false-positive rate, false discovery rate, false-negative rate, accuracy, F1 Score, and Matthews Correlation Coefficient with values) were exceeded by the proposed model, which achieved a $0.9880,0.9892,0.9891,0.9882,0.0108,0.0109,0.0120,0.9886,0.9885$, and 0.9772 , respectively. This work demonstrated the utility and benefits of applying transfer learning in conjunction with loss as an SVM. It has been determined that combining transfer learning approaches with deep learning algorithms enhances the accuracy of pneumonia diagnosis and prediction while also significantly reducing the detection time.

\section{Declarations}

\section{Acknowledgements}

We would like to thank the editor and anonymous reviewers for their helpful comments and valuable suggestions.

\section{Authors' contributions}

All authors take part in the discussion of the work described in this paper. Esraa Hassan, and Samir Elmougy conceived and designed the experiments; Mahmoud Y. Shams, Esraa Hassan, and Noha A. Hikal performed the experiments Esraa Hassan, and Samir Elmougy analyzed the data; Mahmoud Y. Shams, Esraa Hassan, and Samir Elmougy wrote the paper.

\section{Availability of data}

https://github.com/ncbi-nlp/COVID-19-CT-CXR

\section{Ethics approval and consent to participate}

Not applicable.

\section{Consent for publication}


Not applicable.

\section{Competing interests}

The author declares that they have no competing interests.

\section{Author details}

${ }^{1}$ Faculty of Artificial Intelligence, Kafrelsheikh University, 33511, Egypt

${ }^{2}$ Dept. of Information Technology, Faculty of Computers and Information, Mansoura University, Mansoura, 35516, Egypt

${ }^{3}$ Dept. of Computer Science, Faculty of Computers and Information, Mansoura University, Mansoura, 35516, Egypt

*Correspondence should be addressed to esraa.hassan@ai.kfs.edu.eg

\section{Publisher's Note}

Springer Nature remains neutral with regard to jurisdictional claims in published maps and institutional affiliations.

\section{References}

1. A.J. Jinia et al., Review of Sterilization Techniques for Medical and Personal Protective Equipment Contaminated with SARS-CoV-2. IEEE Access 8, 111347-111354 (2020). doi:10.1109/ACCESS.2020.3002886

2. E. Hassan, M. Shams, N.A. Hikal, S. Elmougy, “Plant Seedlings Classification using Transfer," no. July, pp. 3-4, 2021

3. S. Elmuogy, N.A. Hikal, E. Hassan, "An efficient technique for CT scan images classification of COVID-19," vol. 40, pp. 5225-5238, 2021, doi: 10.3233/JIFS-201985

4. S. Minaee, R. Kafieh, M. Sonka, S. Yazdani, G. Jamalipour, “Medical Image Analysis,” pp. 1-9, 2021

5. C. Zheng et al., “Deep Learning-based Detection for COVID-19 from Chest CT using Weak Label,” pp. 1-13, 2020, doi: 10.1101/2020.03.12.20027185

6. K. Lensink et al., "Segmentation of Pulmonary Opacification in Chest CT Scans of COVID-19 Patients," 2020, [Online]. Available: http://arxiv.org/abs/2007.03643

7. M. Cherti, J. Jitsev, "Effect of large-scale pre-training on full and few-shot transfer learning for natural and medical images," pp. 1-18, 2021, [Online]. Available: http://arxiv.org/abs/2106.00116

8. N.K. Chowdhury, M.A. Kabir, M.M. Rahman, N. Rezoana, "ECOVNet: An Ensemble of Deep Convolutional Neural Networks Based on EfficientNet to Detect COVID-19 From Chest X-rays," 2020, doi: 10.7717/peerj-cs.551

9. O. Shahid, M. Nasajpour, S. Pouriyeh, R.M. Parizi, M. Han, M. Valero, Machine Learning Research Towards Combating COVID-19: Virus Detection (Spread Prevention, and Medical Assistance, 2003)

10. A. Vijayalakshmi, R. Kanna, B, “Deep learning approach to detect malaria from microscopic images. Multimed. Tools Appl. 79, no. 21-22 (2020). doi:10.1007/s11042-019-7162-y. ,", , pp. 15297-15317

11. M. Baruch, "Fighting COVID-19 in the Dark: Methodology for Improved Inference Using Homomorphically Encrypted DNN."

12. X. Mei et al., Artificial intelligence-enabled rapid diagnosis of patients with COVID-19. Nat. Med. 26(8), 1224-1228 (2020). doi:10.1038/s41591-020-0931-3

13. U. Maheen, K.I. Malik, G. Ali, "Comparative Analysis of Deep Learning Algorithms for Classification of COVID-19 X-Ray Images," vol. 9, no. 4, pp. 73-81, 2021 
14. H. Zhang et al., Automated detection and quantification of COVID-19 pneumonia: CT imaging analysis by a deep learning-based software. Eur. J. Nucl. Med. Mol. Imaging 47(11), 2525-2532 (2020). doi:10.1007/s00259-020-049531

15. P. Kumar, S. Kumari, Detection of coronavirus Disease (COVID-19) based on Deep Features. no. March (2020). doi:10.20944/preprints202003.0300.v1

16. H. Gunraj, L. Wang, A. Wong, X.-Ray “Chest, Images, ” Front. Med. 7, 1-12 (2020). doi:10.3389/fmed.2020.608525

17. A. Sarkar, J. Vandenhirtz, J. Nagy, D. Bacsa, M. Riley, Identification of Images of COVID-19 from Chest X-rays Using Deep Learning: Comparing COGNEX VisionPro Deep Learning 1.0 $0^{\mathrm{TM}}$ Software with Open Source Convolutional Neural Networks. SN Comput. Sci. 2(3), 1-18 (2021). doi:10.1007/s42979-021-00496-w

18. R. Harkness, G. Hall, A.F. Frangi, N. Ravikumar, K. Zucker, "The pitfalls of using open data to develop deep learning solutions for COVID-19 detection in chest X-rays."

19. P. Sarosh, S.A. Parah, R.F. Mansur, G.M. Bhat, "Artificial Intelligence for COVID-19 Detection-A state-of-the-art review," Arxiv, vol. 2, no. November 2020, 2020

20. A. Ebadi, P. Xi, A. Maclean, S. Tremblay, S. Kohli, A. Wong, “COVIDx-US - An Open-Access Benchmark Dataset of Ultrasound Imaging Data for AI-Driven COVID-19 Analytics," pp. 1-12, 2021

\section{Figures}

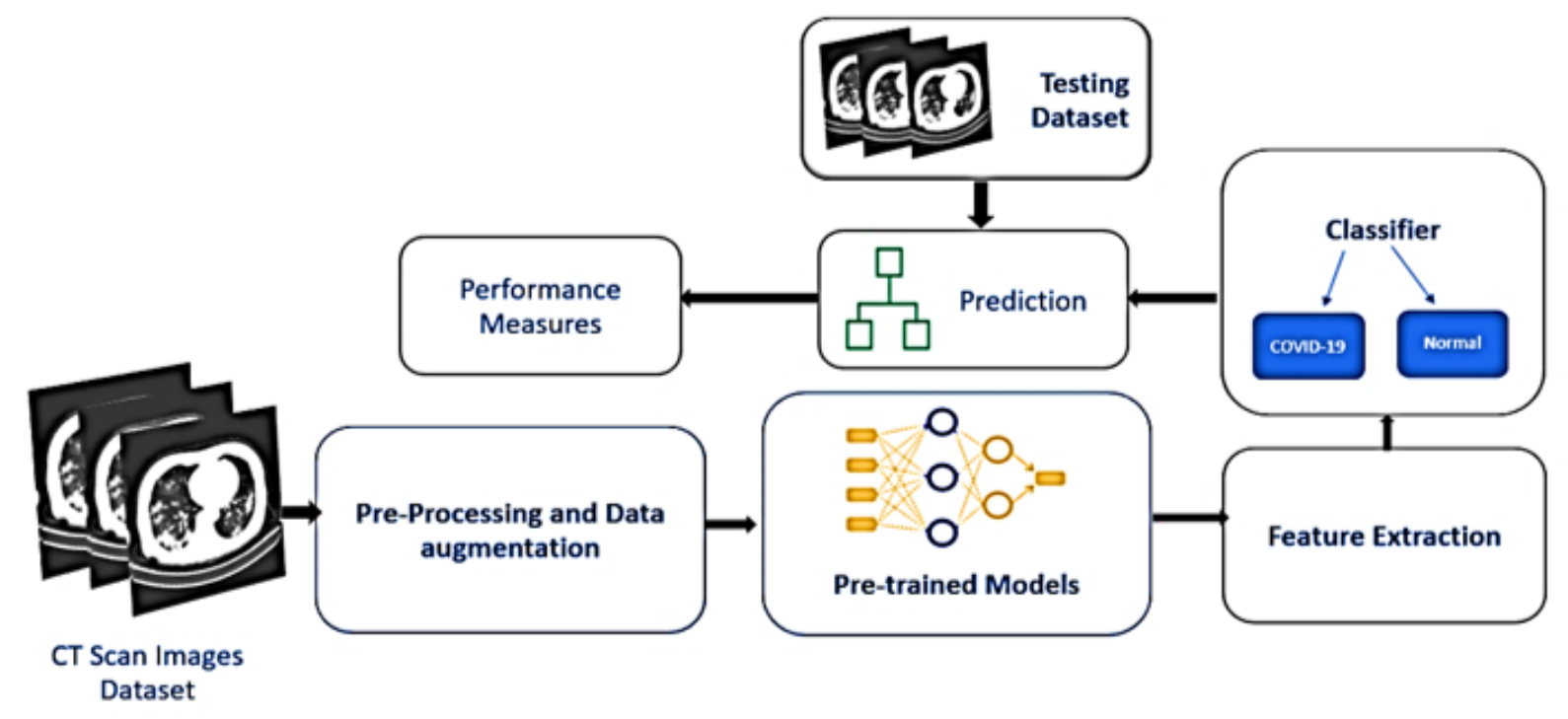

Figure 1

The proposed architecture for CT Scan images 


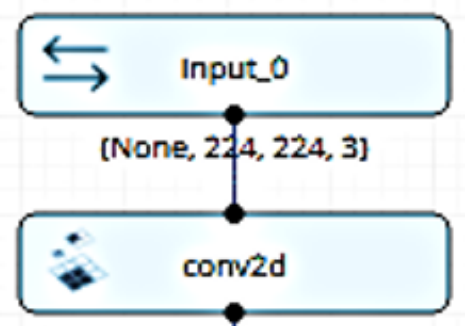

(None, $224,224,64$ )

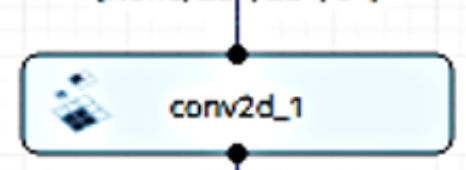

(None, 22f, 224, 64)

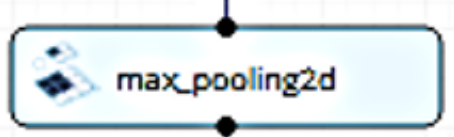

(None, 11/, 112, 64)

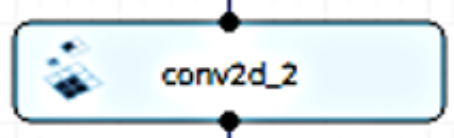

(None, 112, 112, 32)

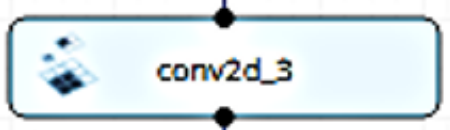

(None, 112, 112, 32)
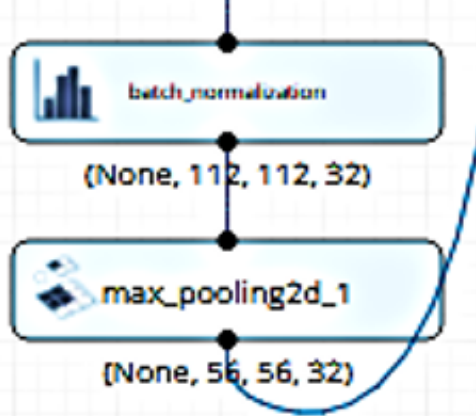

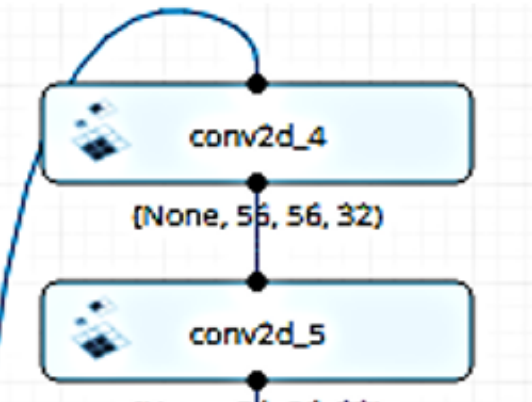

(None, $51,56,32$ )

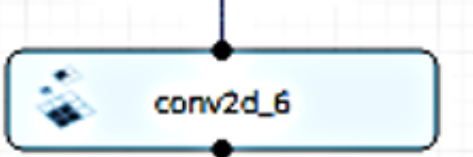

(None, 5 , 56, 32)

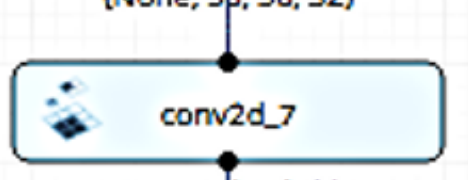

(None, 5 , 56, 32)

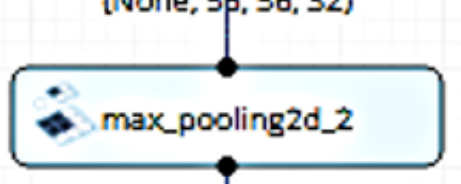

(None, $23,28,32$ )

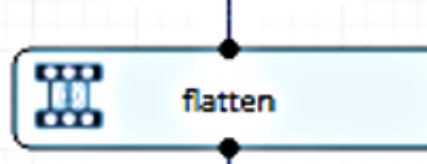

[None, 25088]

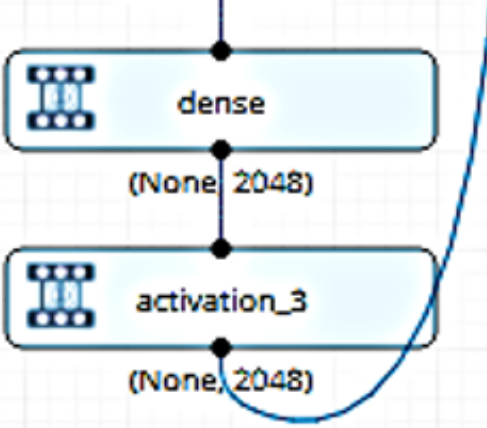

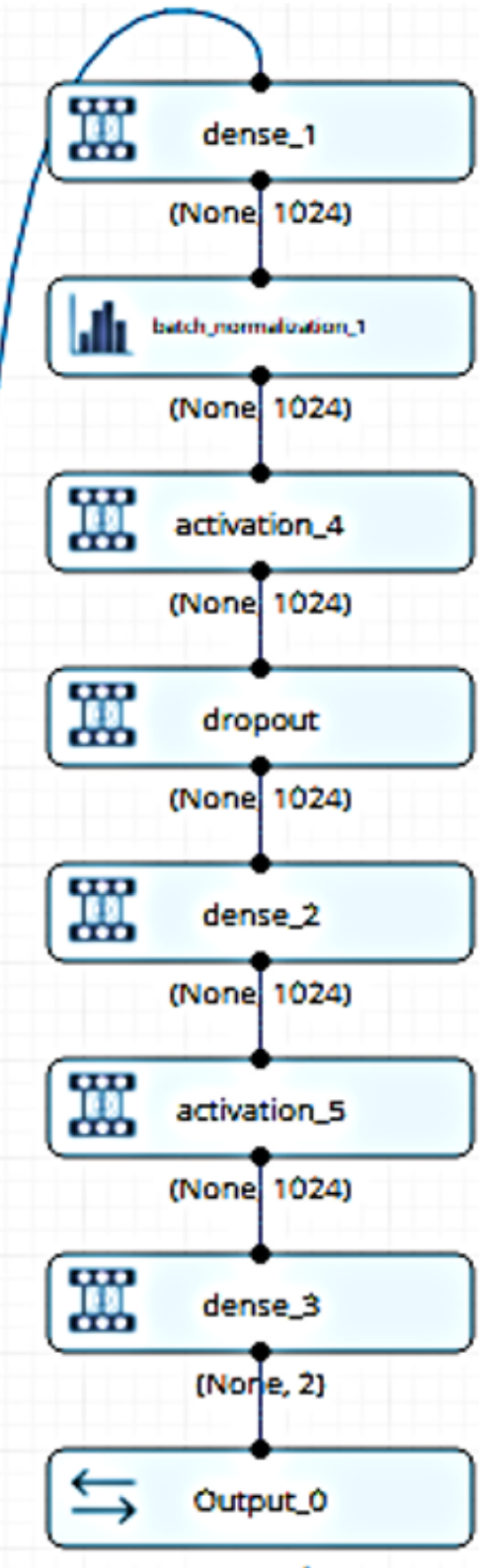

[None, 2]

Figure 2

The main CDNN proposed model layers
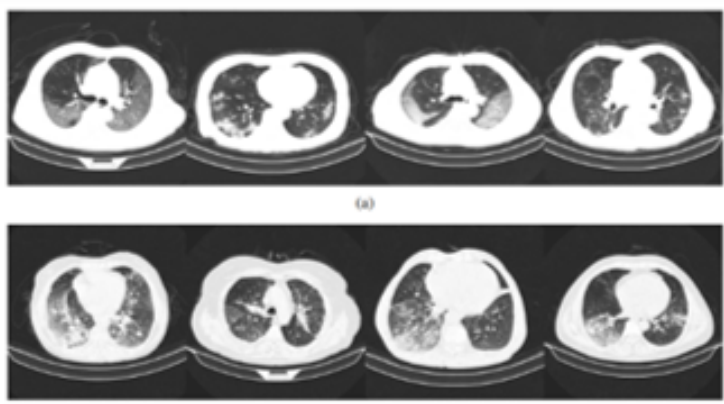


\section{Figure 3}

Example chest CT images from the COVIDx-CT dataset, and illustrating (a) COVID-19
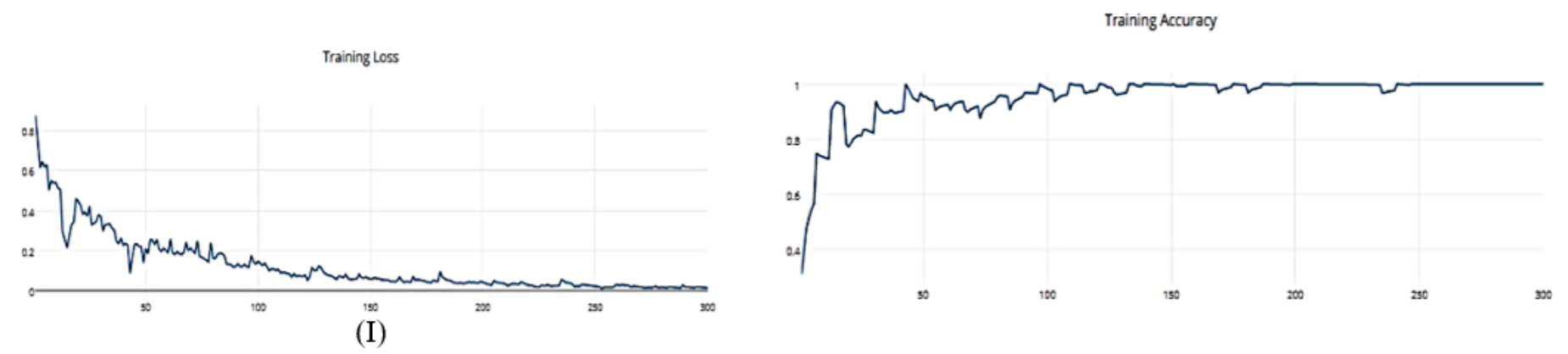

(II)

\section{Figure 4}

(I) The Training accuracy value of CDNN model, (II) The Loss value of CDNN model (SGD)

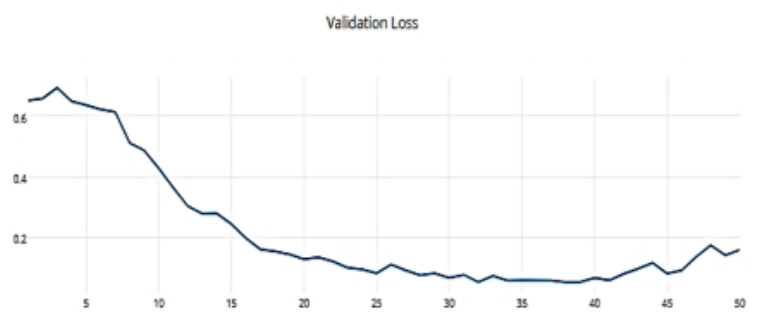

(I)

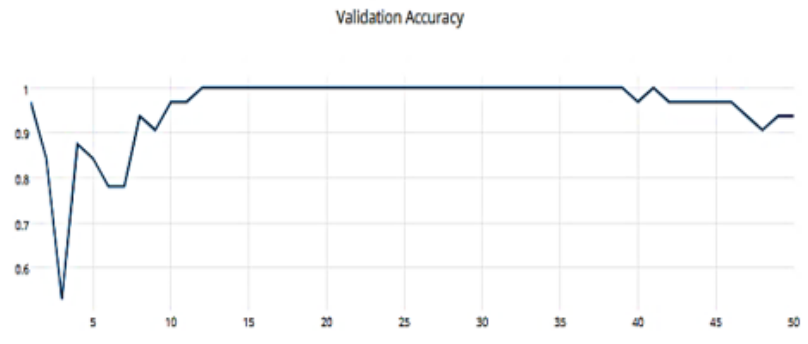

(II)

\section{Figure 5}

(I) The Training accuracy value of CDNN model, (II) The Loss value of CDNN model (Adam)

Training Loss

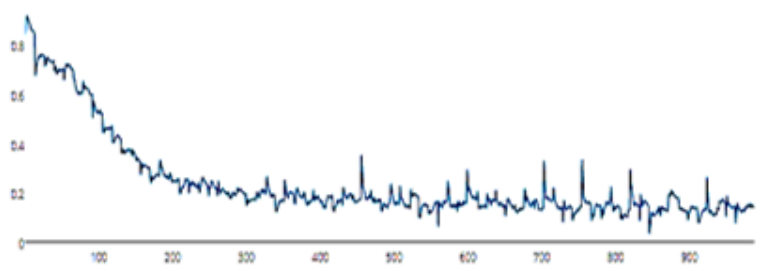

(I)

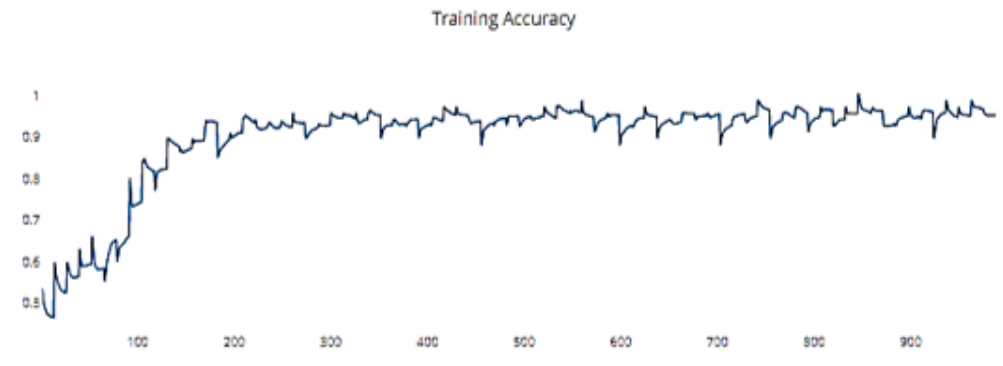

(II)

Figure 6 
(I) The Training accuracy value of CDNN model, (II) The Loss value of CDNN model (SGD)

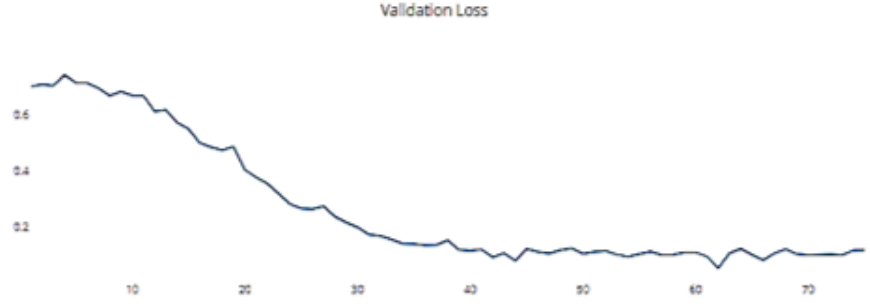

(I)

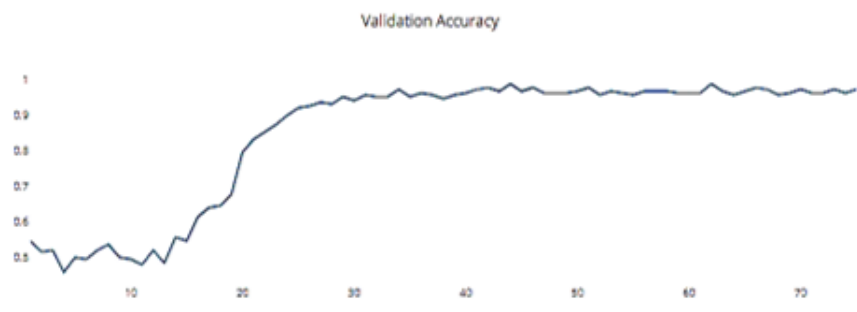

(II)

\section{Figure 7}

(I) The Training accuracy value of CDNN model, (II) The Loss value of CDNN model (SGD)

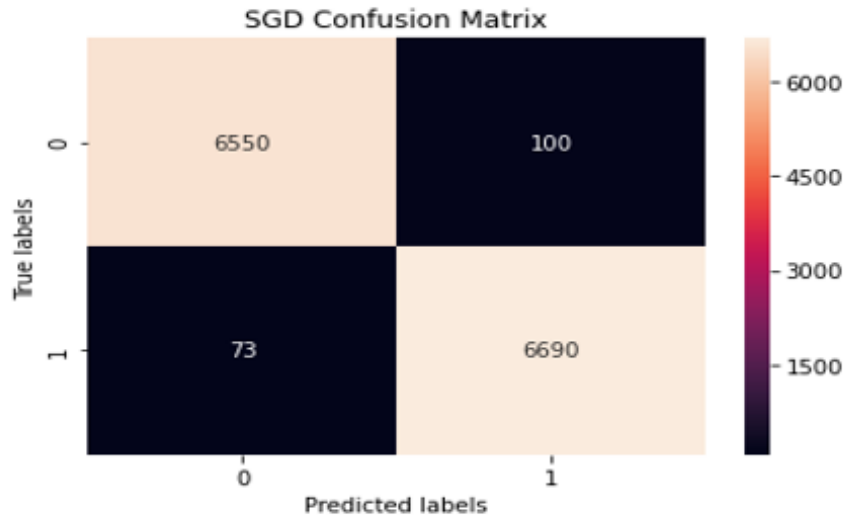

(I)

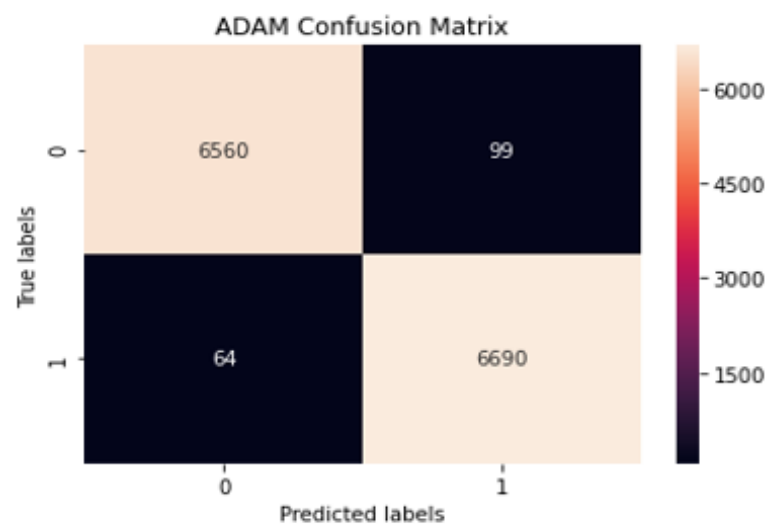

(II)

\section{Figure 8}

(I) The confusion matrixes for SGD (Training stage), (II) The confusion matrixes for Adam (Training stage) 


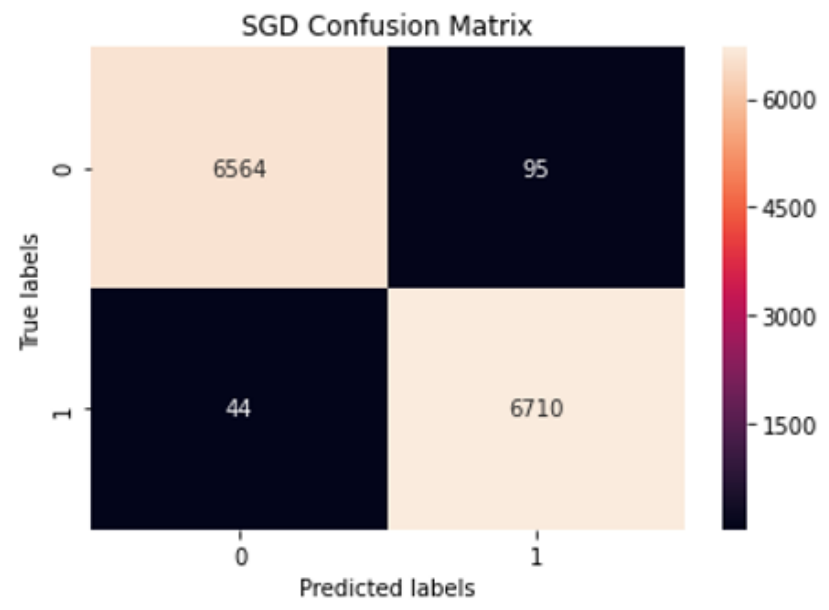

(I)

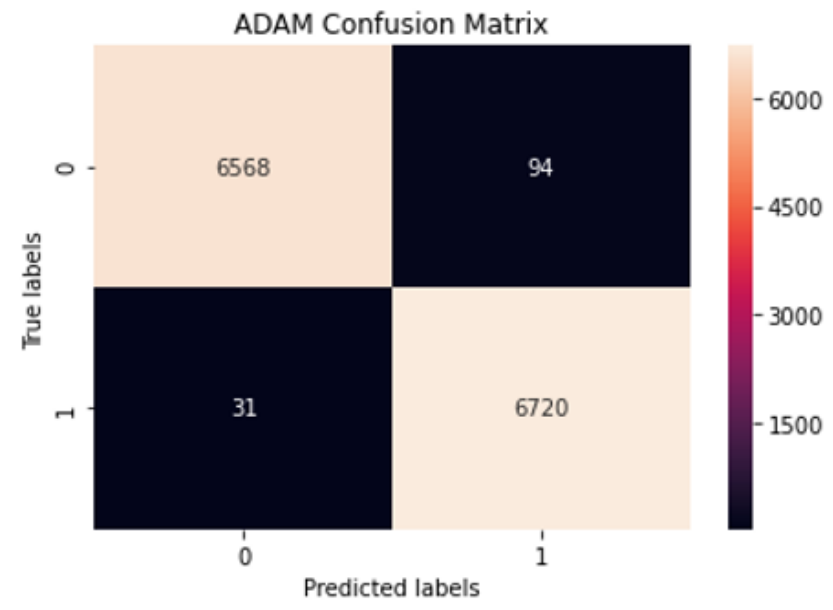

(II)

\section{Figure 9}

(I) The confusion matrixes for SGD (Testing stage), (II) The confusion matrixes for Adam (Testing stage) 